\title{
TNOVACIÓN SOCIAL Y SU IMPORTANCIA EN LA GESTIÓN DEL CONOCIMIENTO Y LA PARTICIPACIÓN CIUDADANA*
}

Social innovation and its importance in knowledge management and citizen participation

(ID) Carlos Elías Rincón**

(ID) Diana Patricia Hermith ${ }^{\star \star *}$

(ID) Wilson Bautista Molina****

* El artículo nace del Centro de Innovación Educativa en Ingeniería (CIEI)

** Ingeniero de sistemas y computación. Magíster en Technology and Innovation Management. Docente en la Universidad Autónoma de Occidente, Cali - Colombia. Email: cerincon@uao.edu.co

*** Bióloga molecular. Magíser en Ingeniería. PhD in Engineering and Science of Information. Investigadora Postdoctoral en la Pontificia Universidad Javeriana, Cali - Colombia. Email: dphermith@javerianacali.edu.co

**** Artista Plástico, Licenciado en Educación Artística. Docente en Fundación Universitaria Católica Lumen Gentium, Cali - Colombia. Email: wbautista@unicatolica.edu.co

Fecha de recepción: 21 de junio del 2017

Fecha de aprobación: 3 de noviembre del 2017

\section{Cómo citar / How to cite}

Rincón, C. E.; Hermith, D. P. y Bautista Molina, W. (2018). Innovación social y su importancia en la gestión del conocimiento y la participación ciudadana. trilogía Ciencia Tecnología Sociedad, 10(18), 51-61. 
Resumen: la innovación social, como motor para el desarrollo de la sociedad en ciencia y tecnología, se aborda en esta reflexión desde dos perspectivas analíticas: la gestión del conocimiento y la participación ciudadana. Este artículo plantea la necesidad de considerar dichas líneas conceptuales para entender a profundidad la relación intrínseca que existe entre proyectos creativos de innovación social, en donde no solo se solucionan problemas específicos, pero también incitan a la inclusión y acción colectiva, dejando en su ejecución un gran rastro de conocimiento nuevo generado, esencial para contribuir en un impacto real en la sociedad o comunidad objetivo. Se realizó una revisión detallada, buscando indicios de actitudes colaborativas, cuya construcción de valores sea para el bien común en democrática estabilidad en comunidades vulnerables buscando innovar durante el abordaje de problemas sociales complejos. Este artículo también busca profundizar en conceptos de innovación ciudadana, sociedades del conocimiento y su aporte en la ciencia, tecnología y sociedad.

Palabras clave: innovación social, gestión del conocimiento, participación ciudadana, sociedades del conocimiento.

Abstract: Innovation promotes social development in science and technology. This reflection paper addresses it from two analytical perspectives: knowledge management and citizen participation. These two concepts must be considered to fully understand the intrinsic relationship among creative social innovation projects. The latter do not only solve specific problems, but also encourage collective action and inclusion. During their implementation, a great deal new of knowledge is generated, and this is essential to make a real impact on society or a target community. A detailed review was conducted to find signs of collaborative attitudes that foster values for the common good in democratic stability of communities seeking to innovate while solving complex social issues. This article aims to delve into the concepts of social innovation, knowledge societies and their contribution to science, technology and society.

Keywords: social innovation, knowledge management, citizen participation, knowledge societies. 


\section{INTRODUCCIÓN}

La innovación social es un concepto abordado de múltiples formas y varía según el contexto y país donde se discute, muchas veces se hace énfasis en un enfoque comercial, dejando de lado sutilmente la importancia por la cual se está realizando esta clasificación, la cual es el beneficio directo y evidente en las personas necesitadas, un ejemplo de esto es la definición hecha por la Oficina de Consejeros en Política Europea (BEPA, por sus siglas en inglés) considerando las innovaciones sociales comonuevas ideas, productos, servicios, modelos de negocio, que simultáneamente satisfacen las necesidades del mercado como las de la sociedad (BEPA, 2011). Sin embargo, pareciera que este concepto estuviese incompleto, pues hay autores que profundizan en este tema de una forma más adecuada, como Geoff Mulgan, quién menciona que la innovación social se refiere a actividades innovadoras que fueron diseñadas con el objetivo de satisfacer una necesidad social a través de la inclusión de la propia comunidad afectada, los resultados de estos servicios son predominantemente ejercidos a través de organizaciones cuyos propósitos primarios son sociales (Mulgan, 2006).

Siguiendo con esta línea de pensamiento, la innovación social se deriva de la percepción de una necesidad social insatisfecha y, por tanto, trabajar hacia una mejora en las condiciones de la misma. Así, pues, los valores son una parte intrínseca en la motivación de alcanzar la innovación social; entre otras incluye aumentar la participación democrática, reducir la pobreza, mejorar las condiciones de los discapacitados, contribuir a ciudades ambientalmente y socialmente más sostenibles, inclusive el aumento de la capacidad de los sobrevivientes de cáncer para apoyarse mutuamente, entre otros (Andrew, Klein y Mohamoud, 2010).

En síntesis, estas definiciones sugieren que los ciudadanos pasan de ser receptores pasivos de acciones institucionales, a convertirse en protagonistas y productores de soluciones mediante un proceso de apropiación y empoderamiento de las problemáticas sociales, lo que en otras palabras se conoce participación ciudadana activa. Esto es de vital importancia cuando se considera la magnitud y complejidad de los problemas relacionados con el sistema de salud, transporte, pobreza, desnutrición, delincuencia común y bajos niveles de educación en zonas rurales.

Los procesos de innovación, como parte del desarrollo social y empresarial, traen consigo un conjunto de «reglas» tácitas que pueden afectar en sí mismo el avance de estos, enmarcándose ya no en un problema de actividades o habilidades técnicas, sino en una cuestión de gestión del conocimiento representado actitudes, valores, tradición cultural, sentido común y entendimiento profundo de la comunidad vulnerable con la que se está trabajando, entonces se podría concluir que implícitamente la innovación social requiere de procesos de cambio de paradigmas y nuevos retos en investigación en sus proyectos de gestión.

En este sentido, se han seleccionado dos líneas analíticas para encontrar qué tipo de conexiones conceptuales permiten la construcción en procesos de innovación social desde la gestión del conocimiento y la participación ciudadana en asuntos de ciencia y tecnología. Cada una de estas perspectivas se aborda procurando evidenciar aquellas dinámicas que potencian procesos de innovación de base científico tecnológica, a partir de un trabajo colaborativo y de una participación activa de la ciudadanía. También se describe la metodología utilizada en la investigación y finalmente, se enuncian las conclusiones.

\section{METODOLOGÍA}

Se realizó una revisión bibliográfica de publicaciones científicas, con el objetivo de identificar aquellos aspectos y preocupaciones que existen en el contexto de la innovación social. Como se mencionó previamente, en esta exploración se 
INNOVACIÓN SOCIAL Y SU IMPORTANCIA EN

LA GESTIÓN DEL CONOCIMIENTO Y LA PARTICIPACIÓN CIUDADANA

consideraron dos perspectivas distintas: gestión del conocimiento y la participación ciudadana. El proceso de recopilación y síntesis realizado, desde un enfoque conceptual y metodológico, permite abordar la innovación social como constante explícita para el desarrollo de la sociedad basado en el conocimiento y la colaboración.

Estos dos enfoques seleccionados establecen un marco de referencia para el estudio y profundización en los conceptos de innovación social, buscando comprender su dinámica e impacto en la toma de decisiones que trascienden la sociedad junto con su capacidad innovativa para resolver problemas que aquejan a comunidades vulnerables y necesitadas.

\section{RESULTADOS Y DISCUSIÓN}

Por décadas, el Gobierno Nacional colombiano le ha apostado a la ciencia, tecnología y la innovación, como base fundamental para lograr la competitividad del país, reconociendo que es precisamente la producción, difusión y uso del conocimiento lo que permitirá lograr una transformación productiva y social, sin mencionar por supuesto la inclusión de forma activa de los ciudadanos en la resolución de problemáticas sociales. Considerando esto y entre los resultados de la revisión bibliográfica, se evaluó de una forma critica las conexiones entre innovación social y la gestión del conocimiento, encontrando por ejemplo la trascendencia y evolución a sociedades del conocimiento que impulsan a la innovación ciudadana de una forma clara y evidente. Otro resultado es la importancia de la transformación de comunidades vulnerables que se logra solo con su participación activa en esfuerzos para desarrollar soluciones nuevas con alto potencial innovador que a su vez resuelven una problemática local que los aquejan. En ese orden de ideas las siguientes secciones pretenden ofrecer un análisis crítico desde cada una de las perspectivas planteadas.

\section{Perspectiva de la innovación social desde la gestión del conocimiento}

Para empezar, la gestión del conocimiento, junto con sus procesos de generación y transferencia de la información, enfatizan en la valoración del capital intelectual de una empresa (Know-How) para maximizar y potencializar su aplicación en procesos de mejoramiento continuo de la organización (Schalk, 2014). Este concepto está muy orientado comercialmente a una manera de mejorar la colaboración institucional, evitar repetir errores y heredar conocimiento de un empleado a otro. Una premisa emerge: será posible replicar las características de la gestión del conocimiento en proyectos de innovación social en la medida que el impacto en poblaciones vulnerables sea mayor, con esfuerzos y actividades menos repetitivas, pero más informadas de las problemáticas a resolver, quizá lográndolo por aquella transferencia de información y aprendizaje activo que busca la gestión del conocimiento.

Es precisamente en estas definiciones donde la relación entre la gestión del conocimiento y la innovación social podrían complementarse un poco, en la medida en que la primera busca el avance empresarial; y la segunda un impacto social sostenible. Un ejemplo de esto es RodríguezGómez y Gairín (2015), los cuales plantean que la gestión del conocimiento se percibe como un capital o activos socialmente construidos, incitando así a la innovación y aprendizaje que desencadenan cambios organizacionales, donde las empresas tienen la oportunidad de aprovechar el conocimiento disponible para actuar de forma rápida y eficiente ante un mercado variable y necesidades sociales cada vez más complejas (Jiménez-Narváez, 2005), o en este caso, la preparación para intervenir en problemas de interés social.

En adición a esto, las actividades de la gestión del conocimiento en los proyectos de desarrollo local 
conforman un proceso social complejo, con unas características propias que emergen de cada cada territorio, con circunstancias y condiciones en que solo los actores locales que intervienen en su aplicación conocen en profundidad la situación. Su contribución es esencial por la naturaleza estratégica del manejo de la información, relevante para la resolución de problemas exclusivos de la comunidad (Castillo, Santana y González, 2017).

Ya que se toca el tema de la información, cabe realizar la aclaración de que tanto la gestión de la información como la del conocimiento tienen varios elementos en común, sin embargo, la manera en que se desarrollan es diferente. La gestión del conocimiento busca cubrir las necesidades de todos los miembros de la organización, para capturar el conocimiento empírico de la empresa y compartirlo tanto en el ámbito interno como externo, de forma que se genere nuevo conocimiento basado en el previo; mientras que la gestión de la información se enfoca en los datos estructurados de la organización, más que en sus capacidades y procesos mecánicos y/o sistematizados (Bustelo y Amarilla-Iglesias, 2001). Por tanto, las organizaciones que consideran el conocimiento como un recurso estratégico, involucran al individuo como miembro y componente básico para lograr aprendizajes que generan capital social, intelectual y estructural para la transformación del valor de los servicios que ofrece la empresa misma.

Por otro lado, para poder incentivar la gestión del conocimiento, el contexto educativo es sumamente relevante, apoyando iniciativas de formación docente interdisciplinares, flexibles y participativas; coherentes por supuesto, con los propósitos y la naturaleza de esta cultura tecnocientífica, donde la colaboración interdisciplinar es clave para el desarrollo de la sociedad en todos sus niveles, tales como el económico, educativo, cultural, entre otros. «Crear conocimiento consiste en llegar a una nueva relación entre conocimientos previos que abre nuevas vías de solución o exploración. Esta 'nueva relación' es el producto de la creatividad, de la imaginación» (Darceles,
2007, p. 100), en ese orden de ideas, el saber común, las creencias, la tradición oral y escrita son también fuente de creatividad social.

Con esto en mente, el énfasis en innovación social es muy pertinente pues la generación de conocimiento, pues también se considera como una fuente principal de riqueza y bienestar de una nación, de manera que los contextos científicos y tecnológicos hacen parte de la organización política y de la educación. Por ejemplo, en España se creó la «Ley Orgánica de Universidades», que permite que la ciencia básica de estudiantes universitarios se vincule a las empresas para generar nuevo conocimiento e innovación tecnológica (Quintanilla, 2007), logrando así un esfuerzo claro y visible por parte del Gobierno en conectar gestión de conocimiento e impacto social.

También se habla de la construcción de una «sociedad del conocimiento», concepto profundizado por la Organización de las Naciones Unidas para la Educación, la Ciencia y la Cultura (UNESCO) en el 2005, donde revela que comprende distintas actividades comerciales, sociales y culturales que sirven, a su vez, como insumos para la creación de nuevas soluciones, aprendizaje y prosperidad de las comunidades involucradas. Es así como el conocimiento y sus esfuerzos para la innovación a nivel social, forman un componente importante en la producción que dirige a la economía y desarrollo de un país. Como resultado, es evidente que la innovación social y la gestión del conocimiento van ligadas no solo en virtud del mejoramiento continuo de comunidades vulnerables, sino de facilitar el aprendizaje y la solución de problemas sociales.

En contraste, la investigación realizada por Palacio (2011), donde hace una descomposición del término «sociedades del conocimiento», de su significado por partes y concluye, entre otras cosas, que existe una conexión importante entre los problemas sociales y las acciones relacionadas con el conocimiento. Este vínculo implícito se le denomina 
competitividad, que a su vez es un índice de logro de apropiación de conocimiento, crecimiento económico y generación de empleo. Sin embargo, no existe una única sociedad del conocimiento, sino unas «sociedades del conocimiento», las cuales no se centran únicamente en la competitividad, sino en la cultura, estabilización de las familias (Vrcelj y Zloković, 2014), el desarrollo sostenible (Dattakumar, Chong, Malone, Sharma y Valenzuela, 2016) y en prácticas sociales. Así pues, la competitividad no es necesariamente índice de apropiación social de conocimiento, pero también lo es el aprovechamiento de las tradiciones culturales, creencias e incremento de la participación de los distintos miembros de las comunidades relacionadas (Olivé, 2012).

En el contexto colombiano, el nuevo desafío de la innovación social es evitar que espacios lingüísticos y culturales colectivos queden excluidos en proyectos de desarrollo de comunidades vulnerables, por ello radica la importancia de que este conocimiento local y cultural sirva de insumo esencial, para que estimule la participación ciudadana como un mecanismo para apoyar procesos de resolución de sus respectivos problemas sociales. Esto pareciera una tarea poco sencilla, si se considera la infraestructura necesaria para lograrlo, debido a que es costoso llevar tecnología (computadores, internet, redes eléctricas, etc.) al campo y zonas rurales, pues aún hace falta mejorar la infraestructura de telecomunicaciones en estas ubicaciones geográficas (Barón y Gómez, 2012).

Es así como la Cumbre Mundial, de la Organización de las Naciones Unidas (ONU), describe que cualquier persona debe poseer un espacio de capacidades TIC lo suficientemente amplio para poder utilizarlo (Echeverría, 2008) y ser un miembro activo en la gestión del conocimiento y la innovación. Sin embargo, estas capacidades varían entre países y regiones, quizá la brecha problemática se podría reducir a través de la educación, para fomentar el aprendizaje y un mejor uso de los medios informáticos (Gordillo, 2005).
En adición a todo esto, se encontró que en el evento «Colombia: diálogo cultural y Tecnologías de Información y Comunicación para el fortalecimiento de los procesos comunitarios», se identificaron ejes de reflexión en torno a las alianzas, la apropiación del conocimiento, la formación, procesos organizativos y de dirección, que aportan a la calidad de vida de las comunidades, en temas relacionados con la salud, educación, derechos humanos, recursos naturales, entre otros (Ceballos, 2005). En el documento se destacan la innovación social con diez principios del trabajo colaborativo en red divididos en sinergia, legitimidad, escala, cooperación, creatividad, oportunidades, diversidad, motivación, tecnologías y actores. Aunque el trabajo colaborativo en red es solo una de las estrategias posibles para procesos de innovación social, marca la pauta para forjar las bases de la gestión del conocimiento.

En contraste, algunos pensadores consideran que la tecnología deshumaniza y es destructiva, por ejemplo, este es el caso de los deterministas tecnológicos pesimistas: «La tecnología, dicen estos, determina irresistiblemente nuestras vidas, pero al hacerlo nos deshumaniza, nos aliena en nuestra relación con la naturaleza y destruye el medio ambiente» (Borgmann, 2005, p. 83), mientras otros piensan que la tecnología no es ni buena ni mala; simplemente es parte del desarrollo y de las creaciones de los seres humanos. Sin embargo, la premisa se mantiene, cómo orientar el uso de las TIC para fomentar la innovación ciudadana y sus respectivas sociedades del conocimiento.

Además, el uso de las tecnologías de la información y de la comunicación está cambiando notablemente las conductas, comportamientos y rutinas diarias de la sociedad, creando tendencias culturales que influyen entre los principios de la gestión del conocimiento y la innovación social. Así mismo, estas tecnologías brindan oportunidades de nuevos contextos, donde se construye y se enriquece una red de participación de procesos de innovación ciudadana (Rodríguez-Gómez y Gairín, 2015). 
Adicionalmente, para profundizar un poco más en esta línea de pensamiento, los autores Atuesta-Venegas, Ceballos-Moncada y Alvis (2016) comparten una asociación interesante entre tecnologías de la información y de la comunicación con el concepto de la gestión del conocimiento, donde surgen distintas dinámicas colaborativas y procesos de co-creación, con posibilidades de vinculación de métodos artesanales, tradición cultural, folclor y estrategias aplicadas para la solución de desafíos de interés común. En otras palabras, la colaboración en medio del trabajo interdisciplinar coordinado de manera conjunta para estimular la reflexión y el uso de espacios de aprendizaje, que sirven como insumo a proyectos de innovación social.

Recapitulando, se puede resumir que la gestión del conocimiento tiene un potencial enorme para la transformación de la sociedad. Su conexión implícita con la innovación social en temas concernientes a la educación, sostenibilidad y cambios empresariales, abren la puerta a la mayor potencialidad de una organización, la cual está fundamentada en la capacidad de aprendizaje colectivo.

\section{Perspectiva de la innovación social desde la participación ciudadana}

La innovación social significa el establecimiento de nuevos vínculos y nuevas relaciones que ayudan a superar las líneas de fragmentación donde predomina la exclusión social. La innovación social promueve espacios de reinserción en el mercado laboral y en la política, a través de la participación activa de los diversos actores involucrados, sean los prestadores de servicios o sean la población objetivo (Herrera, 2015, p. 65).

Si bien es claro que los problemas sociales relacionados con la salud, pobreza, desnutrición, delincuencia común, educación y transporte son caracterizados por su alto grado de complejidad, existen varios actores que colaboran en conjunto, proponiendo soluciones y proyectos de carácter social como lo son universidades, empresas y funcionarios públicos, quienes interactúan constantemente en un flujo de decisiones y convenios, que dan como resultado el desarrollo científico, económico, tecnológico y social de un país. Su trabajo colaborativo incentiva cambios en las políticas públicas que permite que proliferen mecanismos participativos que catapultan el auge de la innovación social (Todt, 2006).

Esto invita a la participación y consenso en distintos campos donde se ofrezca un espacio para encontrar soluciones a diversas problemáticas; dónde se identifiquen y se propongan los mecanismos creativos que permitan potenciar procesos de participación ciudadana. Según (Ziman, 2003) estamos involucrados en una sociedad pluralista, donde nacen instituciones autónomas que producen conocimiento científico y especializado en una gran diversidad de contextos sociales. Inclusive, la participación pública implica su evolución constante, con sus diversas formas de pensar y de actuar, con el interés de resolver en forma conjunta los problemas sociales, abriendo así la oportunidad de capitalizar en el conocimiento generado (Do Nascimento, 2005).

Por otro lado, vale la pena resaltar la diferencia entre democracia participativa y participación ciudadana. En la primera, el pueblo no solo elige sus representantes por medio del voto, sino que tiene la posibilidad de intervenir directamente en la toma de ciertas decisiones de carácter político, como por ejemplo el plebiscito, referendo, audiencias públicas y otros mecanismos que permiten al ciudadano influir en el rumbo del país de forma democrática (Alfredo, 2016). Sin embargo, este artículo se enfoca más en la línea analítica donde participación ciudadana es orientada a la acción colectiva, entendida como nuevas iniciativas de actuar de los individuos de la sociedad en pro de la resolución de sus propios problemas, explorando maneras de cooperación y comunicación, buscando atender la resolución de los conflictos que surgen en sus respectivas comunidades (Estensoro y Zurbano, 2010). 
INNOVACIÓN SOCIAL Y SU IMPORTANCIA EN

LA GESTIÓN DEL CONOCIMIENTO Y LA PARTICIPACIÓN CIUDADANA

No obstante, la alianza de las organizaciones responsables de las políticas públicas, de las empresas creadoras de riqueza y de las universidades como generadoras de conocimiento debería convertirse en un instrumento privilegiado para un desarrollo social, científico y tecnológico, de naturaleza responsable y colaborativa, en el que se propicien acuerdos, sinergias y metas compartidas para el beneficio individual y colectivo (RodríguezGómez y Gairín, 2015). El desarrollo y progreso sostenible, involucra a todas las instituciones de la sociedad, sin cuyo compromiso resulta difícil abordar nuevos retos. Por tanto, en los procesos de innovación social, dónde se podría extender el crecimiento y el desarrollo de un país.

Tal es el caso del Programa Nacional de Cuidados Domiciliarios (PNDC), que consiste en ayudar a la población adulta mayor por medio de la capacitación de cuidadores y servicios de asistencia socio-sanitaria, esto es posible a través del apoyo de Gobierno Nacional, convenios con departamentos, municipios y provincias, junto con las cooperativas de trabajo de todo el país (Fassio, et al., 2015). En efecto, la forma como este programa se ha desplegado en el suelo colombiano deja entrever una posible innovación social por dos razones: primero, el empoderamiento de la comunidad, PNDC busca transformar personas naturales entre los 25 y 50 años sin trabajo, en cuidadores con entrenamiento en atención primaria y especializada en adultos mayores. Segundo, resuelve una necesidad social creciente, pues muchos ancianos (edad de 75 años o más) presentan dependencia de terceros para realizar las actividades básicas e instrumentales de la vida cotidiana, que sin su ayuda la calidad de vida y salud podrían verse deterioradas rápidamente.

Dentro del contexto de la participación ciudadana, se sugiere pensar el progreso científico y tecnológico de manera responsable y vincular a la ética, puesto que no todo lo científicamente posible y tecnológicamente viable es necesariamente deseable o admisible (Quintanilla, 2007). Un claro ejemplo de esto es México, donde se ha reconocido la necesidad de actuar en contra de la brecha entre el desarrollo social y el tecnológico, con el objetivo de incrementar la productividad y el bienestar en línea con la competitividad y la globalización.

Esto abre un camino potencial hacia una mejor calidad de vida, en un contexto mucho más amplio y equilibrado, con un buen ejemplo evidenciado en el caso de estudio planteado en López y González (2003), donde la comunidad desde su experiencia de vida con el medio ambiente y su formación básica promedio reflexiona y participa activamente en la manifestación pública de sus intereses de colectividad. Este es un caso que demuestra cómo una comunidad comprometida y activa logra apropiarse de un problema y encuentra formas conjuntas para resolverlo. Otro buen ejemplo de participación ciudadana para la innovación social son los Living Labs en Europa, los cuales consisten en redes de conocimiento y de prácticas sociales (Finquelievich, 2007). Aquí, la investigación y la creatividad están centradas en el usuario y la idea principal se basa en involucrar a éste último como eje central en el proceso de innovación social.

Por tanto, resulta imperativo promover la participación ciudadana en temas que estimulen la innovación social, empoderando a las personas para que pasen de ser receptores pasivos de una problemática a ciudadanos activos en su solución. Los ejemplos mencionados anteriormente muestran claramente cómo la inclusión de comunidades vulnerables en el despliegue de proyectos de innovación social logra permitir que la solución planteada no solo sea efectiva sino sostenible a largo plazo. Se podría concluir, entonces, que cuando un Gobierno logra brindarle a un ciudadano educación y herramientas necesarias, esta persona estará más dispuesta a pagar impuestos, a cumplir con las leyes y a trabajar colaborativamente en resolver sus propias problemáticas locales. 


\section{CONCLUSIONES}

La innovación social se considera en esta revisión como eje central del desarrollo de la sociedad. Considerando así la gestión del conocimiento en la colaboración entre distintos campos como el científico, tecnológico, social y empresarial, para ofrecer espacios que propicien la creación de soluciones a diversas problemáticas y mecanismos; mientras que simultáneamente apoyen los procesos de participación y acuerdo ciudadano. Sin esta perspectiva, no se puede pretender potenciar la eficiencia económica dejando de lado comunidades vulnerables con necesidades reales y complejas (Pascual, 2008).

Primero, la innovación social se convierte en una meta $u$ objetivo a alcanzar, que emerge de esfuerzos de participación ciudadana en la resolución de problemáticas locales relacionadas con la salud, pobreza, desnutrición, delincuencia común, educación, etc. Lo cierto es que no se puede pretender ejecutar proyectos de interés social sin considerar la colaboración voluntaria y acción colectiva de la comunidad objetivo, es de esta forma que el desconocimiento de los actores involucrados en las distintas problemáticas mencionadas anteriormente es una falla evidente en cualquier proyecto de desarrollo (Lora y Rocha, 2016). Se concluye entonces que se busca promover la innovación en comunidades formadas por personas que interactúan entre sí, que tienen una cultura y un lenguaje en común, ofreciéndoles las herramientas necesarias para tomar acción en medio de su propia situación local.

Segundo, la relación entre innovación social y la gestión del conocimiento pareciera ser un poco compleja, pues el conocimiento puede surgir de otras fuentes diferentes a las científicas, como es el caso del conocimiento tradicional y ancestral, como también el llamado conocimiento tácito proveniente de costumbres urbanas, la herencia cultural oral, hasta la mitología misma compartida de generación en generación (Lora y Rocha, 2016). Esta complejidad es clara en la medida que se profundiza y evoluciona en sociedades del conocimiento, donde el aprendizaje y aprovechamiento de la cultura, tradiciones y el sentido común hace la diferencia en las distintas soluciones que se proponen para resolver problemáticas de interés ciudadano.

Finalmente, la innovación social es considerada por su trascendencia en la participación consciente y responsable para la transformación sociocultural de comunidades en medio de la intervención de sus problemáticas. Así pues, se plantea la necesidad de crear espacios concertados, que permitan construir una ideología central, capaz de prescribir un claro papel social para las ciencias y sus tecnologías asociadas. Esta revisión ha abordado una reflexión que profundiza en dos perspectivas, que de alguna forma proponen generar y propiciar la participación ciudadana y la labor educativa de la gestión del conocimiento, con la expectativa de acudir a proyectos interdisciplinarios que le permiten a la sociedad en general, impulsar el aprendizaje en virtud del bien común y de la construcción de nuevas soluciones con potencial innovador.

\section{REFERENCIAS}

Alfredo, R. N. (2016). Participación ciudadana e interpretación de la constitución. Análisis de la jurisprudencia de la Corte Constitucional colombiana en materia de democracia participativa. Revista IUS, (37), 171.

Andrew, C., Klein, J. y Mohamoud, H. (2010). Social innovation. Montreal: CRISES, Centre de recherche sur les innovations sociales.

Atuesta-Venegas, M. R., Ceballos-Moncada, A. F. y Alvis, R. G. (2016). Co-creación como metodología para la Apropiación Social de la Ciencia y la Tecnología (ASCYT) del recurso agua. Caso Urabá-Antioqueño- Colombia. El Ágora USB, 16(1), 277-286.

Barón, L. F. y Gómez, R. (2012). De la infraestructura a la apropiación social: panorama sobre las políticas de las Tecnologías de Información 
INNOVACIÓN SOCIAL Y SU IMPORTANCIA EN

LA GESTIÓN DEL CONOCIMIENTO Y LA PARTICIPACIÓN CIUDADANA

y Comunicación (TIC) en Colombia. Signo y Pensamiento, 31(61), 38-55.

BEPA. (2011). Empowering people, driving change: social innovation in the European Union France. Bureau of European Policy Advisers (BEPA).

Borgmann, A. (2005). La tecnología y la búsqueda de la felicidad. Revista Iberoamericana de Ciencia Tecnología y Sociedad, 2(5), 81-93.

Bustelo-Ruesta, C. y Amarilla-Iglesias, R. (2001). Gestión del conocimiento y gestión de la información. Boletín del Instituto Andaluz de Patrimonio Histórico, Año VIII(34), 226-230. INFORAREA S. L. España.

Castillo-Arzola, N.; Santana-Cruz, J. V. y GonzálezVillafruela, M. R. (2017). Impacto social de la gestión del conocimiento en el desarrollo municipal. Revista trilogía. Ciencia, Tecnología, Sociedad, 9(16), 47-61.

Ceballos-Schaulsohn, F. (2005). Diez razones para trabajar en red. Colombia: diálogo cultural y tecnologías de información y comunicación para el fortalecimiento de los procesos comunitarios. Grupo de investigación INFORCAUCA. Colombia.

Darceles-Tife, M. (2007). Gestión del conocimiento y creatividad. Revista EAN, Norteamérica, (61), 97102.

Dattakumar, A.; Chong, G.; Malone, L.; Sharma, R.; Valenzuela, J. (2016). IEEE International Conference on Industrial Engineering and Engineering Management (IEEM), Industrial Engineering and Engineering Management (IEEM), 2016 IEEE International Conference on, 681. doi:10.1109/ IEEM.2016.7797962

Do Nascimento, J. B. (2005). Estrategias de valor para la creación de conocimiento en organizaciones intensivas en conocimiento. Revista Galega de Economía, 14(001-002), 1-19. ISSN: 1132-2799. España.

Echeverría, J. (2008). Apropiación social de las tecnologías de la información y la comunicación.
Revista Iberoamericana de Ciencia, Tecnología y Sociedad CTS, 4(10), 171-182.

Estensoro, M. y Zurbano, M. (2010). Innovación social en la gobernanza territorial. Los casos de Innobasque y de las redes comarcales de la Comunidad Autónoma del País Vasco. EKONOMIAZ, 74, 131-159.

Fassio, A. et al., (2015). Innovación social, políticas públicas y aprendizaje organizacional: el programa nacional de cuidados domiciliarios. TRILOGÍA. Ciencia. Tecnología y Sociedad, 7(13), 9-24.

Finquelievich, S. (2007). Innovación, tecnología y prácticas sociales en las ciudades: hacia los laboratorios vivientes. Revista Iberoamericana de Ciencia, Tecnología y Sociedad CTS, 3(09), 135-152.

Gordillo, M. (2005). Cultura científicay participación ciudadana: materiales para la educación CTS. Revista Iberoamericana de Ciencia, Tecnología y Sociedad, 2(6), 123-135.

Herrera, P. M. (2015). Bonos de impacto social: de la innovación social a la innovación financiera responsable. TRILOGÍA. Ciencia. Tecnología y Sociedad, 7(13), 63-73.

Jiménez-Narváez, L. (2005). Modelización sistémica de la innovación y del aprendizaje tecnológico. Innovar, 15(25), 81-89.

López-Cerezo, J. y González-García, M. (2003). Interfaces percepción-participación-regulación en la dinámica de las políticas públicas sobre ciencia, tecnología y medio ambiente. Revista Iberoamericana de Ciencia, Tecnología y Sociedad CTS, septiembre, 73-84.

Lora, P. y Rocha, D. (2016). Promoción de la innovación social a través de la utilización de metodologías participativas en la gestión del conocimiento. Equidad \& Desarrollo, (25), 159178.

Mulgan, G. (2006). The process of social innovation. innovations, 1(2), 145-162. 
Olivé, L. (2012). Sociedades del conocimiento justas, democráticas y plurales en América Latina. Pensamiento y Cultura, 15(1), 5-19.

Palacio-Sierra, M. (2011). Construcción de la sociedad del conocimiento y las políticas públicas de apropiación social de la ciencia, la tecnología y la innovación. TRILOGÍA. Ciencia. Tecnología y Sociedad, 5, 17-39.

Pascual-Trillo, J. (2008). La insostenibilidad como punto de partida del desarrollo sostenible. Revista Iberoamericana de Ciencia, Tecnología y Sociedad CTS, 04(11), 81-94.

Quintanilla, M. (2007). La investigación en la sociedad del conocimiento. Revista Iberoamericana de Ciencia, Tecnología y Sociedad CTS, 03(8), 183-194.

Rodríguez-Gómez, D. y Gairín-Sallán, J. (2015). Innovación, aprendizaje organizativo y gestión del conocimiento en las instituciones educativas. Educación, 24(46), 73-90.
Schalk Quintanar, A. (2014). Modelo de enseñanza-aprendizaje para adultos en la era del conocimiento. Diseño y estructura del modelo. Gestión en el Tercer Milenio, 8(16), 6387.

Todt, O. (2006). La gobernanza tecnocientífica en la Unión Europea. Revista Iberoamericana de Ciencia, Tecnología y Sociedad CTS, 03(7), 21-42.

UNESCO. (2005). Hacia las sociedades del conocimiento. Paris, Francia: Unesco.

Vrcelj, S. y Zloković, J. (2014). Knowledge Society and the (De)Stabilization of Families. Procedia - Social and Behavioral Sciences, 159(5th World Conference on Psychology, Counseling and Guidance, WCPCG-2014, 1-3 May 2014, Dubrovnik, Croatia), 537-540. doi:10.1016/j. sbspro.2014.12.420

Ziman, J. (2003). Ciencia y sociedad civil. Revista Iberoamericana de Ciencia, Tecnología y Sociedad CTS, 1(1), 177-188. 